\title{
Relationship of promising methods in the detection of anthracycline-induced cardiotoxicity in breast cancer patients
}

\author{
Ben F. Bulten ${ }^{1,2}$ (1) - Hein J. Verberne ${ }^{3}$ Louise Bellersen ${ }^{4}$ Wim J. G. Oyen ${ }^{2} \cdot$ \\ Aida Sabaté-Llobera $^{2,5}$ • Annelies M. C. Mavinkurve-Groothuis ${ }^{6}$ Livia Kapusta $^{7,8}$. \\ Hanneke W. M. van Laarhoven ${ }^{9,10} \cdot$ Lioe-Fee de Geus-Oei ${ }^{1,2,11}$
}

Received: 10 July 2015 / Accepted: 10 September 2015 / Published online: 23 September 2015

(c) The Author(s) 2015. This article is published with open access at Springerlink.com

\begin{abstract}
Purpose It remains challenging to identify patients at risk of anthracycline-induced cardiotoxicity. To better understand the different risk-stratifying approaches, we evaluated ${ }^{123}$ I-metaiodobenzylguanidine $\quad\left({ }^{123} \mathrm{I}-\mathrm{mIBG}\right)$ scintigraphy and its interrelationship with conventional echocardiography, 2D strain imaging and several biomarkers.

Methods We performed ${ }^{123} \mathrm{I}-m \mathrm{IBG}$ scintigraphy, conventional and strain echocardiography and biomarker (NTproBNP, TNF- $\alpha$, galectin-3, IL-6, troponin I, ST-2 and sFlt-1) assessment in 59 breast cancer survivors 1 year after anthracycline treatment. Interobserver and intermethod variability was calculated on planar and SPECT ${ }^{123} \mathrm{I}-m \mathrm{IBG}$ scintigraphy, using the heart/mediastinum $(\mathrm{H} / \mathrm{M})$ ratio and washout (WO). Pearson's $r$ and multivariate analyses were performed to identify correlations and independent predictors of ${ }^{123} \mathrm{I}-m \mathrm{IBG}$ scintigraphy results.
\end{abstract}

Ben F. Bulten

benbulten@gmail.com

1 MIRA Institute for Biomedical Technology and Technical Medicine, University of Twente, Zuidhorst Room 2.65, Drienerlolaan 5, PO Box 217, 7500 AE Enschede, The Netherlands

2 Department of Radiology and Nuclear Medicine, Radboudumc, Geert Grooteplein 10, Nijmegen, The Netherlands

3 Department of Nuclear Medicine, Academic Medical Center, Meibergdreef 9, Amsterdam, The Netherlands

4 Department of Cardiology, Radboudumc, Geert Grooteplein 10, Nijmegen, The Netherlands

5 Department of Nuclear Medicine, Hospital Universitari de Bellvitge, Feixa Larga, Barcelona, Spain
Results Delayed planar anterior whole-heart ROI (WH) $\mathrm{H} / \mathrm{M}$ ratios and WO were the most robust ${ }^{123} \mathrm{I}-m \mathrm{IBG}$ parameters. Significant correlations were observed between ${ }^{123}$ I- $m$ IBG parameters and several conventional echo parameters, global longitudinal and radial strain (GLS and GRS) and galectin-3. The highest Pearson's $r$ was observed between delayed H/M ratio and GRS (Pearson's $r$ 0.36, $p=0.01)$. Multivariate analysis showed that GRS was the only independent predictor of the delayed $\mathrm{WH} \mathrm{H} / \mathrm{M}$ ratio $(p=0.023)$.

Conclusion The delayed planar H/M ratio is the most robust ${ }^{123} \mathrm{I}-m \mathrm{IBG}$ parameter. It correlates with several conventional echocardiographic parameters, GLS, GRS and galectin-3. Of these, only GRS predicts the H/M ratio.

Keywords Breast cancer - Anthracyclines . Cardiotoxicity $\cdot 2 \mathrm{D}$ strain imaging $\cdot{ }^{123} \mathrm{I}-\mathrm{mIBG}$ scintigraphy $\cdot$ Biomarkers

6 Princess Maxima Center for Pediatric Oncology, Lundlaan 6, Utrecht, The Netherlands

7 Department of Children's Heart Center, Radboudumc, Geert Grooteplein 10, Nijmegen, The Netherlands

8 Pediatric Cardiology Unit, Tel Aviv Sourasky Medical Center, Weizmann Street 10, Tel Aviv, Israel

9 Department of Medical Oncology, Academic Medical Center, Meibergdreef 9, Amsterdam, The Netherlands

10 Department of Medical Oncology, Radboudumc, Geert Grooteplein 10, Nijmegen, The Netherlands

11 Section of Nuclear Medicine, Department of Radiology, Leiden University Medical Center, Albinusdreef 2, Leiden, The Netherlands 


\section{Introduction}

Anthracyclines are widely used for (neo)adjuvant treatment of breast cancer [11,27]. This class of drugs is associated with cardiotoxicity [3]. Anthracycline-induced cardiotoxicity (AIC) can be acute, which may lead to chemotherapeutic dose reduction, but is generally reversible. However, development of chronic AIC (i.e. $\geq 1$ year after therapy) is often irreversible and may have a significant impact on the overall prognosis and survival of breast cancer survivors [37]. As treatment with anthracyclines resulted in significantly improved breast cancer survival over the past decades, the importance of (early) detection and prevention of potential side effects has increased. Most commonly, the possible deleterious effects of anthracyclines on left ventricular function are monitored by left ventricle ejection fraction (LVEF) measurement using multigated radionuclide angiography (MUGA) or (2D non-contrast) echocardiography $[3,17]$. However, the reproducibility of echocardiography parameters varies and both techniques only detect LVEF changes that occur after considerable damage has been acquired [19, 36]. An adequate technique to identify patients at risk of cardiotoxicity (i.e. before the damage occurs) is still lacking [17].

The pathophysiology of AIC is complex and not yet fully understood. Recent research has focused on the topoisomerase-II $\beta$ enzyme as the core defect mechanism, which is believed to induce cell death upon the formation of a complex with the anthracycline doxorubicin [24, 38]. This topoisomerase-II $\beta$-doxorubicin complex-induced cell death subsequently triggers a cascade of cytokine release and compensatory mechanisms, which are potential targets for early detection of AIC [7]. When myocytes die, cardiac output declines, with the release of norepinephrine (NE) in the synapse by the sympathetic nervous system as one of the first neurohumoral responses [1]. The release of NE in combination with a decreased presynaptic NE reuptake (i.e. NE transporter downregulation) leads to an increased concentration of NE in the synaptic cleft $[12,15]$. Metaiodobenzylguanidine ( $m \mathrm{IBG}$ ) is an analogue of the sympathetic neurotransmitter NE. Labelling of $m$ IBG with ${ }^{123} \mathrm{I}$ allows for scintigraphic assessment of sympathetic activity and may provide a measure for early detection of AIC [15]. The most commonly used methods to quantify myocardial ${ }^{123} \mathrm{I}-m \mathrm{IBG}$ uptake are the measurement of the heart/mediastinum (H/M) ratio and washout (WO). Increased sympathetic cardiac activity is characterized by a decreased $\mathrm{H} / \mathrm{M}$ ratio and an increased myocardial washout of ${ }^{123} \mathrm{I}-m \mathrm{IBG}$.

Other novel methods that may detect AIC in an early stage include 2D strain (rate) imaging with echocardiography and blood biomarkers. 2D strain (rate) imaging measures the relative deformation (i.e. stretch) of cardiac tissue in three different axes [25]. Since strain can differentiate active from passive movement, subtle regional differences can be detected long before LVEF deteriorates [31]. Blood biomarkers can be measured to provide information on cardiac pathological processes, as summarized in the cytokine hypothesis by Braunwald et al. [7]. Myocyte injury, whether it is due to hemodynamic or ischaemic stress, induces release of the compensatory prohormone N-terminal probrain natriuretic peptide (NT-proBNP), the myofibrillar protein troponin I and different cytokines including tumour necrosis factor alpha (TNF- $\alpha$ ) and interleukin-6 (IL-6). Subsequently, activated monocytes secrete the interleukin-1 receptor family member ST2, while macrophages produce galectin-3 [7]. Furthermore, the angiogenesis promoter-soluble Fms-like tyrosine kinase receptor 1 (sFlt-1) is activated [21].

As ${ }^{123}$ I- $m$ IBG scintigraphy, 2D strain (rate) imaging and blood biomarkers reflect different aspects of the same pathophysiological mechanism, they most likely show some interrelationship. However, knowledge on this possible correlation is still lacking. Therefore, the aim of the current study was to study the relation between ${ }^{123} \mathrm{I}-m \mathrm{IBG}$ scintigraphy, echocardiographic (strain) imaging and a selection of the most promising biomarkers. We aimed to study this in a homogenous group of breast cancer survivors 1 year after a potentially cardiotoxic chemotherapeutic regimen, containing anthracyclines.

\section{Materials and methods}

\section{Patient selection}

All adult female patients presented between October 2010 and May 2012 with breast cancer and at least 1 year after completion of (neo)adjuvant treatment with docetaxel, doxorubicin (i.e. anthracycline) and cyclophosphamide (TAC) were asked to participate in the study. Exclusion criteria consisted of major heart disease (i.e. myocardial infarction, percutaneous coronary intervention or coronary artery bypass graft) at the time of breast cancer diagnosis, renal failure at the time of cardiac evaluation, evidence of breast cancer recurrence or metastatic disease, pregnancy or breast feeding, participation in a research protocol with ionizing radiation 1 year prior to inclusion, diabetes mellitus, Parkinson's disease or an ${ }^{123} \mathrm{I}-\mathrm{m} \mathrm{IBG}$ accumulating tumour.

A detailed medical history and physical examination were obtained in all patients, with special attention to risk factors and signs and symptoms of cardiac disease. Current medication use was noted. A standard 12-lead electrocardiogram was performed and analysed for signs of cardiac disease and rhythm disturbances. The study was approved 
by the medical ethics committee of the Radboud University Medical Center (Nijmegen, The Netherlands), and informed consent was obtained from all patients.

\section{${ }^{123}$ I-mIBG scintigraphy}

Patient medication interfering with ${ }^{123} \mathrm{I}-m \mathrm{IBG}$ uptake was interrupted for at least 5 half-lives after consultation of the attending physician. Thyroid ${ }^{123}$ I uptake was blocked by oral administration of $400 \mathrm{mg}$ potassium perchlorate $1 \mathrm{~h}$ before intravenous injection of $185 \mathrm{MBq}^{123} \mathrm{I}-m \mathrm{IBG}$ (AdreView, GE Healthcare). Patients rested for $30 \mathrm{~min}$ prior to injection.

Planar ${ }^{123}$ I- $m$ IBG images were acquired in anterior and posterior view 15 min ('early') and $4 \mathrm{~h}$ ('delayed') after injection. Imaging was performed during 10 min using a $20 \%$ energy window centred on the $159-\mathrm{keV}$ photopeak of ${ }^{123} \mathrm{I}$, and acquired with a medium energy collimator and stored in a $128 \times 128$ matrix. No scatter correction was applied. Subsequently, a single-photon emission computed tomography (SPECT) of the thorax was performed 35 and $260 \mathrm{~min}$ post-injection, obtaining 32 frames of $60 \mathrm{~s} / \mathrm{frame}$, on a dual-head detector system, using a rotation of $180^{\circ}$.

\section{Measurements on planar images}

On the anterior planar images, 10-20 pixel regions of interest (ROI) were drawn over the upper mediastinum by two observers (BB and ASL) (Fig. 1) [16, 30]. This ROI was then placed over the LV anterior wall to obtain the small left ventricular $(\mathrm{Sm})$ ROI. Furthermore, a whole-heart (WH) ROI was manually determined. Both ROIs were mirrored on the posterior planar images. For all planar images, $\mathrm{H} / \mathrm{M}$ ratios were calculated by dividing the cardiac average counts per pixel by the mediastinal average counts per pixel. Furthermore, a geometric mean of the heart and mediastinum counts was calculated (by means of the formula $\sqrt{\text { countsanterior } \times \text { countsposterior) }}$, resulting in the geo $\mathrm{H} / \mathrm{M}$ ratio. Eventually, this resulted in two measurements of the $\mathrm{H} / \mathrm{M}$ ratios (i.e. anterior and geometric mean) on two time points (i.e. early and delayed) and with two ROI methods (i.e. WH and Sm).

The (background corrected) myocardial WO was defined as described by Veltman [35].

This was done for both the anterior images and the geometric mean method, using both the WH and Sm ROI delineation method. WO is expressed as a percentage.

\section{Single-photon emission computed tomography (SPECT) images}

For SPECT image interpretation, tomographic slices were reconstructed in short axis, horizontal axis and vertical

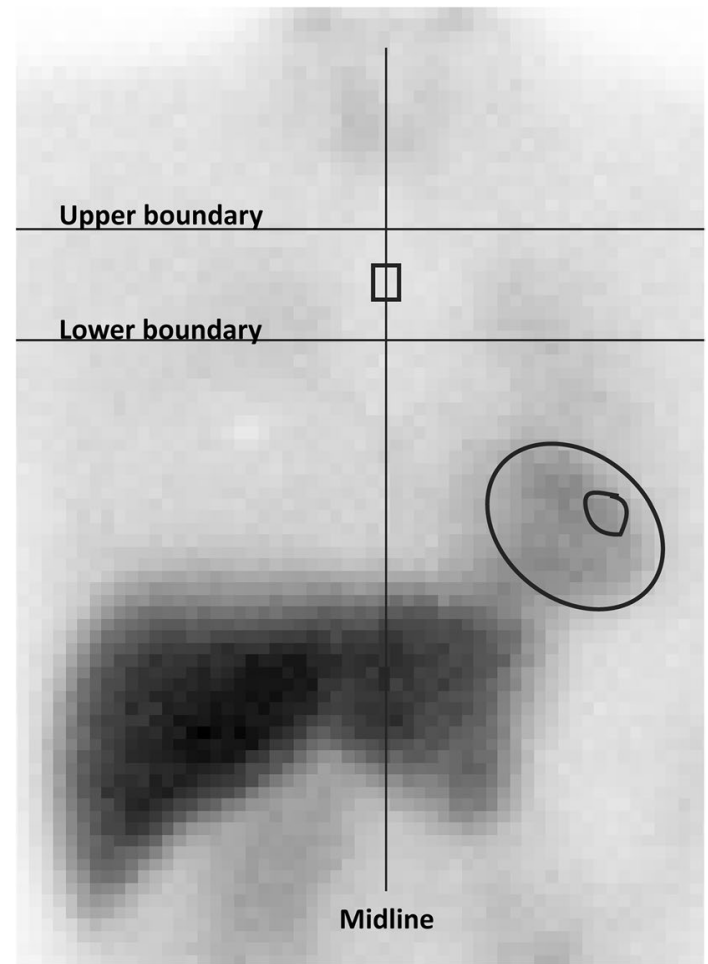

Fig. 1 Standardized approach for the placement of the mediastinal and heart ROIs for H/M ratio determination, adapted from Somsen and Flotats [16, 30] Notice the upper and lower boundary defining the upper mediastinum and the mediastinal midline. The heart ROI consists of either a circular ROI including the left ventricle and the cavum (whole-heart ROI-WH) or a small circular ROI on the left ventricle lateral wall (small LV ROI; Sm)

long axis planes. Early and delayed images were identically aligned so that simultaneous analysis of the image planes was allowed. Mediastinal, WH and LV (i.e. including or excluding the cavum) voxels of interest (VOIs) were visually drawn using Inveon Research Workplace 4.1 (IRW, Siemens Molecular Imaging). The mediastinal VOI had a fixed spherical shape of 20 voxels, and care was taken to exclude thyroid tissue. $\mathrm{H} / \mathrm{M}$ ratios and WO were calculated with mean voxel count as described above.

\section{Echocardiography and biomarkers}

Methods on the measurement of conventional and strain echocardiography parameters and biomarkers have been described extensively elsewhere [9, 34]. The current study focused on the interrelationship of these parameters with ${ }^{123}$ I- $m$ IBG values. The parameters on conventional echocardiography that were studied included the internal dimensions of the left ventricle at end-diastole (LVIDd) and end-systole (LVIDs), the posterior and septal wall thickness at end-diastole (LVPWd, IVSd), left ventricular mass (LVM), left ventricular volume at end-diastole (LVEDV) 
and end-systole (LVESV), left atrial end-diastolic volume (LAEDV), left ventricular ejection fraction (LVEF), early (E) and late (A) diastolic transmitral peak flow velocity (E/A ratio), the systolic to diastolic pulmonary vein peak flow velocity (PV S/D ratio) and early diastolic transmitral peak flow velocity (E) to early diastolic annular velocity $\left(\mathrm{e}^{\prime}\right)$ ratio (E/e' ratio). Measurements of LVEDV, LVESV, LVM and LAEDV were indexed by body surface area (BSA).

The biomarkers we studied were NT-proBNP, TNF- $\alpha$, galectin-3, IL-6, troponin I, ST-2 and sFlt-1. Biochemical risk factors for cardiovascular disease (cholesterol, triglycerides, HDL, LDL, glucose, HbA1C) were also determined.

\section{Statistical analysis}

Patient age and time after treatment are expressed in years or months with range. Other patient characteristics are expressed in numbers of total and percentage. ${ }^{123} \mathrm{I}-m \mathrm{IBG}$ and echocardiographic values are expressed as mean \pm SD. Biochemical values are expressed as median with interquartile range.

Lin's concordance correlation coefficients (LCCs) with $95 \%$ confidence intervals are calculated for interobserver variability and depict the correlation of measurements of two different observers. For clinically relevant agreement, the following criteria are used: LCC values $<0.90$, $0.90-0.95,0.95-0.99$ and $>0.99$ were considered to indicate poor, moderate, substantial and almost perfect agreement, respectively [26]. The coefficient of variation (CV) is the relative ratio of SD to mean and expressed in percentage. $95 \%$ limits of agreement in Bland-Altman plots are defined as mean $\pm 2 \mathrm{SD}$, which is numerically expressed as the coefficient of repeatability (CR; calculated as $1.96 \times \mathrm{SD})$.

Correlations of the various studied methods are expressed in Pearson's $r$. Correlations with a one-tail $p<0.1$ were included in multivariate regression analysis, which was performed in a forward stepwise fashion. Significance was set at $p<0.05$. All statistical analyses were performed with SPSS for Windows, version 20.0.

\section{Results}

\section{Patient characteristics}

Fifty-nine breast cancer survivors were included in the study. All patients had received a full dose of anthracyclines $\left(300 \mathrm{mg} / \mathrm{m}^{2}\right)$, except one who received $250 \mathrm{mg} /$ $\mathrm{m}^{2}$. None of the patients had a history of major cardiac events (i.e. myocardial infarction, percutaneous coronary intervention or coronary artery bypass graft), nor
Table 1 Patient characteristics of the study group $(N=59)$

\begin{tabular}{|c|c|c|}
\hline & $N(\%)$ & Median (range) \\
\hline \multicolumn{3}{|l|}{ Clinical characteristics } \\
\hline Age in years & & $52(36-69)$ \\
\hline Time after treatment in months & & $12.5(10-14)$ \\
\hline \multicolumn{3}{|l|}{ Risk factors } \\
\hline Smoking & $13(22)$ & \\
\hline Hypertension $^{\mathrm{a}}$ & $18(30)$ & \\
\hline Family history ${ }^{\mathrm{b}}$ & $9(16)$ & \\
\hline $\mathrm{HbA} 1 \mathrm{c}>53 \mathrm{mmol} / \mathrm{L}$ & $1(2)$ & \\
\hline $\mathrm{BMI}>30 \mathrm{~kg} / \mathrm{m}^{2}$ & $13(22)$ & \\
\hline \multicolumn{3}{|l|}{ Medication use } \\
\hline ACE/ATII & $2(3)$ & \\
\hline Beta blocker & $6(10)$ & \\
\hline Diuretic & $9(15)$ & \\
\hline Statin & $5(9)$ & \\
\hline Calcium antagonist & $2(3)$ & \\
\hline \multicolumn{3}{|l|}{ Treatment characteristics } \\
\hline \multicolumn{3}{|l|}{ Tumour side } \\
\hline Left & $28(46)$ & \\
\hline Right & $24(39)$ & \\
\hline Both & $11(12)$ & \\
\hline Radiation left thorax side & $22(37)$ & \\
\hline Total radiation in gray & & $65(45-142)$ \\
\hline Cumulative anthracycline dose in $\mathrm{mg} / \mathrm{m}^{2}$ & & $300(250-300)$ \\
\hline
\end{tabular}

a Systolic blood pressure $>140 \mathrm{mmHg}$ and/or use of antihypertensive medication

b Presence of coronary artery disease in a first-degree family member at $<55$ years in men or $<65$ years in women

did any of them indicate chest pain or express heart failure signs/symptoms. One patient presented with a known left bundle branch block. Other patient characteristics, including risk factors and medication use, are displayed in Table 1. One year after treatment the mean LVEF was $62.6( \pm 7)$. LVEF was $<55 \%$ in three patients, respectively, 35, 53 and $54 \%$. The observed LVEF of $35 \%$ was due to aortic sclerosis, which was observed on conventional echocardiography and returned to normal after surgical intervention. The other two patients did not receive follow-up, since they did not meet the criteria for subclinical cardiotoxicity. None of the patients used dexrazoxane.

\section{Planar $\mathrm{H} / \mathrm{M}$ ratio and $\mathrm{WO}$}

Anterior and geometric mean $\mathrm{H} / \mathrm{M}$ ratios and $\mathrm{WO}$ were obtained in all patients on both early and delayed ${ }^{123} \mathrm{I}-m \mathrm{IBG}$ images and by both observers. All anterior $\mathrm{H} / \mathrm{M}$ ratios and WO were significantly lower than geometric mean $\mathrm{H} / \mathrm{M}$ ratios and WO (Table 2). 
Table 2 Planar H/M ratio and WO characteristics

\begin{tabular}{|c|c|c|c|c|c|c|c|c|}
\hline & \multicolumn{3}{|c|}{ Anterior } & \multicolumn{3}{|c|}{ Geometric mean } & \multirow{2}{*}{\multicolumn{2}{|c|}{$\begin{array}{l}\text { Intermethod } \\
\Delta \\
\text { Mean }\end{array}$}} \\
\hline & \multicolumn{2}{|l|}{ Mean } & \multirow{2}{*}{$\begin{array}{l}\text { LCC } \\
95 \% \mathrm{CI}\end{array}$} & \multicolumn{2}{|l|}{ Mean } & \multirow{2}{*}{$\begin{array}{l}\text { LCC } \\
95 \% \text { CI }\end{array}$} & & \\
\hline & $\mathrm{SD}$ & $\mathrm{CV}(\%)$ & & $\mathrm{SD}$ & $\mathrm{CV}(\%)$ & & $\mathrm{SD}$ & $\mathrm{CR}$ \\
\hline \multicolumn{9}{|l|}{$W H$} \\
\hline \multirow[t]{2}{*}{ Early $\mathrm{H} / \mathrm{M}$ ratio } & 2.71 & & 0.90 & 2.99 & & 0.91 & $0.28^{\dagger}$ & \\
\hline & 0.44 & 16.2 & $0.83-0.94$ & 0.40 & 13.4 & $0.85-0.94$ & 0.21 & 0.41 \\
\hline \multirow[t]{2}{*}{ Delayed H/M ratio } & 2.72 & & 0.92 & 2.82 & & 0.92 & $0.10^{\dagger}$ & \\
\hline & 0.51 & 18.8 & $0.86-0.95$ & 0.43 & 15.2 & $0.87-0.95$ & 0.21 & 0.41 \\
\hline \multirow[t]{2}{*}{ WO (\%) } & 21.9 & & 0.83 & 27.5 & & 0.80 & $5.6^{\dagger}$ & \\
\hline & 13.1 & 59.8 & $0.74-0.89$ & 10.2 & 37.1 & $0.69-0.87$ & 5.6 & 11.0 \\
\hline \multicolumn{9}{|l|}{$S m$} \\
\hline \multirow[t]{2}{*}{ Early $\mathrm{H} / \mathrm{M}$ ratio } & 2.80 & & 0.69 & 3.05 & & 0.49 & $0.25^{\dagger}$ & \\
\hline & 0.53 & 18.9 & $0.56-0.78$ & 0.46 & 15.1 & $0.34-0.62$ & 0.24 & 0.48 \\
\hline \multirow[t]{2}{*}{ Delayed H/M ratio } & 2.83 & & 0.71 & 2.92 & & 0.57 & $0.09^{\dagger}$ & \\
\hline & 0.56 & 19.8 & $0.58-0.80$ & 0.47 & 16.1 & $0.40-0.70$ & 0.24 & 0.47 \\
\hline \multirow[t]{2}{*}{ WO $(\%)$} & 20.0 & & 0.53 & 26.0 & & 0.57 & $6.0^{\dagger}$ & \\
\hline & 14.0 & 70.0 & $0.36-0.67$ & 10.7 & 41.1 & $0.37-0.71$ & 7.9 & 15.5 \\
\hline
\end{tabular}

$H / M$ ratio heart/mediastinum ratio, $W O$ washout, $W H$ whole-heart ROI, $S m$ small left ventricle ROI; $S D$ standard deviation, $C V$ coefficient of variation (SD/mean), $L C C$ Lin's correlation coefficient, $C I$ confidence interval, $C R$ coefficient of repeatability $(1.96 \times \mathrm{SD}), \Delta=$ difference

${ }^{\dagger} p<0.001$

\section{Interobserver and intermethod variability}

Interobserver correlations of $\mathrm{WH} \mathrm{H} / \mathrm{M}$ ratios were moderate, of $\mathrm{Sm} \mathrm{H} / \mathrm{M}$ ratios and WO poor (Table 2). However, mean interobserver differences were small for WH H/M ratio (Fig. 2). Intermethod variability, describing the correlation of WH and Sm ROI definition by one observer, demonstrated poor LCCs (Fig. 3).

\section{SPECT $\mathrm{H} / \mathrm{M}$ ratio and $\mathrm{WO}$}

IRW-derived $\mathrm{H} / \mathrm{M}$ ratios are summarized in Table 3. Early $\mathrm{H} / \mathrm{M}$ ratios could be calculated in 52 patients $(88 \%)$, delayed $\mathrm{H} / \mathrm{M}$ ratios in 43 patients $(73 \%)$. This discrepancy was mainly caused by technical or acquisition protocol difficulties (e.g. hardware failure, data file corruption).

\section{Intermethod variability}

The intermethod correlation of SPECT-derived WH versus LV H/M ratio (both early and late) and WO was almost perfect (LCC 0.99). Mean differences were very small (Fig. 4).

\section{SPECT versus planar}

The WH method is the only method used for both planar and SPECT images. Therefore, correlation between SPECT and planar $\mathrm{H} / \mathrm{M}$ ratio was calculated specifically for that method. We observed a Pearson's $r$ of $0.33(p<0.05)$ for the early images, $r=0.35(p<0.05)$ for the late images and $r=0.76(p<0.001)$ for WO.

\section{Conventional and strain (rate) echocardiography and biomarker results}

Although additional data on conventional echocardiographic and strain (rate) imaging and biomarkers are described in earlier work, [9, 34] for the purpose of completeness, an overview of the obtained results is presented in Table 4.

\section{Correlation of ${ }^{123} \mathrm{I}$-mIBG with conventional and strain echocardiography and biomarkers}

Significant correlations between the delayed planar WH H/M ratios and LVEDV/BSA, LVESV/BSA, IVSd, LVM/ BSA, E/A ratio (conventional echocardiography), GLS, GRS (strain echocardiography) and galectin-3 (biomarker) were identified (Table 5). The most significant correlation was observed for GRS (Pearson's $r 0.36, p=0.01$ ), the least significant for E/A ratio (Pearson's $r 0.19, p=0.08$ ). LVEDV/BSA, LVESV/BSA, IVSd, LVM/BSA, GLS and galectin-3 showed an inverse correlation, while E/A ratio and GRS showed a direct correlation. 


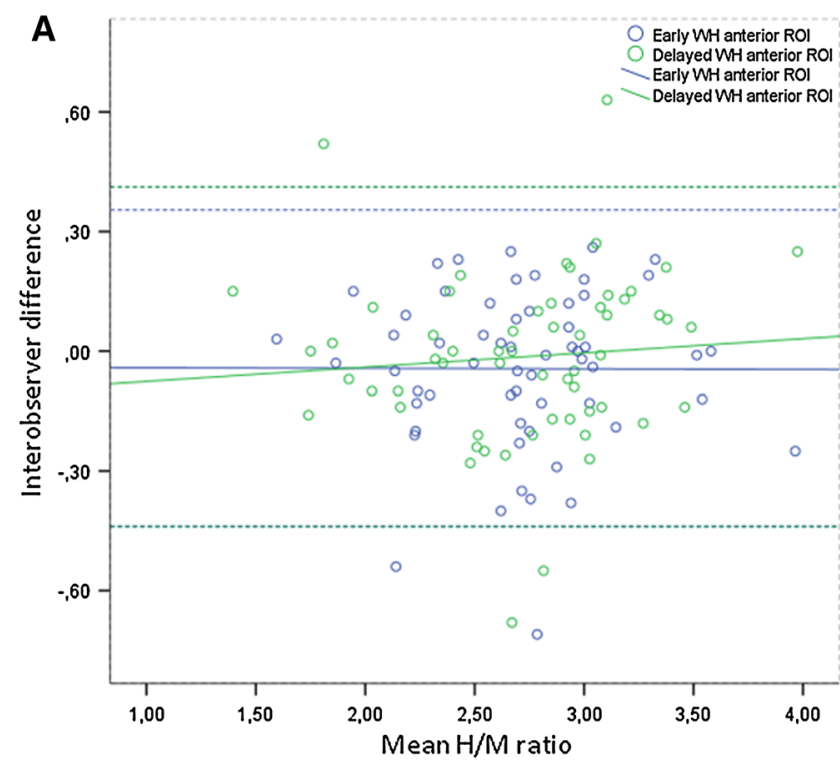

Fig. 2 Bland-Altman plots of interobserver difference versus mean of planar anterior (a) and geometric (b) WH H/M ratio, both on early and on delayed images. Butted lines represent $95 \%$ limits of agreement. A. Mean differences: early images -0.04 (95\% CI -0.10 to

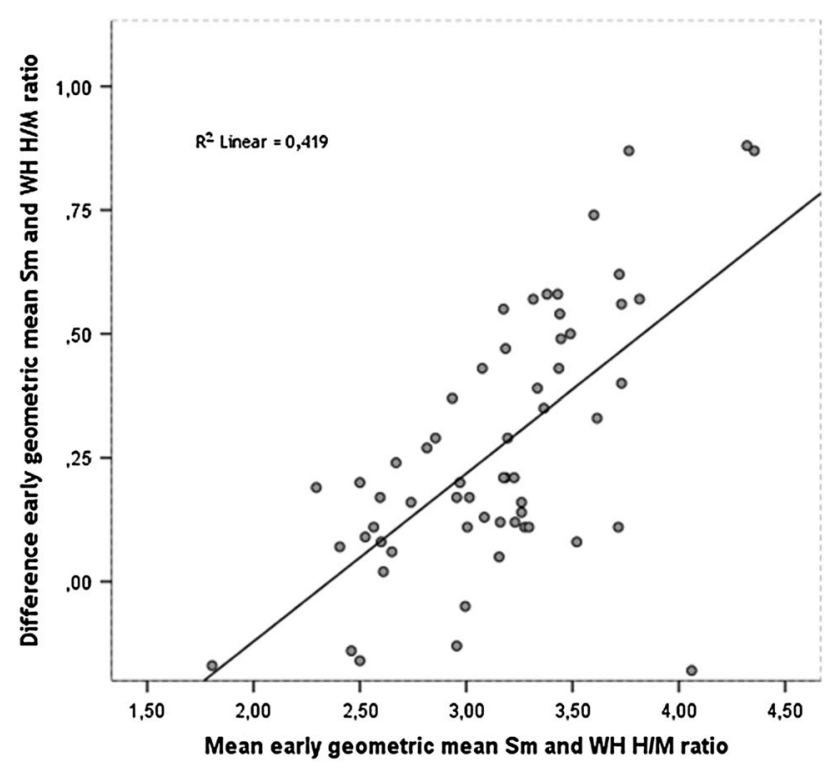

Fig. 3 The dilution effect. Early geo WH versus $\mathrm{Sm} \mathrm{H} / \mathrm{M}$ ratio showed an LCC of 0.87 (95 \% CI 0.81-0.91), $R^{2}=0.42$. The increasing intermethod difference is due to the dilution effect: the difference between cavum and myocardium increases when the myocardium has high ${ }^{123}$ I-mIBG uptake (i.e. the normal heart), and the ROI inclusion of blood pool (i.e. WH ROI) will account more heavily to the average heart count. Other LCCs were 0.79 (95\% CI 0.71-0.86, early anterior), 0.87 (95\% CI 0.81-0.91, delayed anterior) and 0.82 (95\% CI 0.74-0.88, delayed geo). Intermethod analysis of WO showed a poor correlation of 0.82 (95\% CI 0.72-0.88) for anterior images and a moderate correlation of 0.91 (95\% CI 0.85-0.94) for delayed images

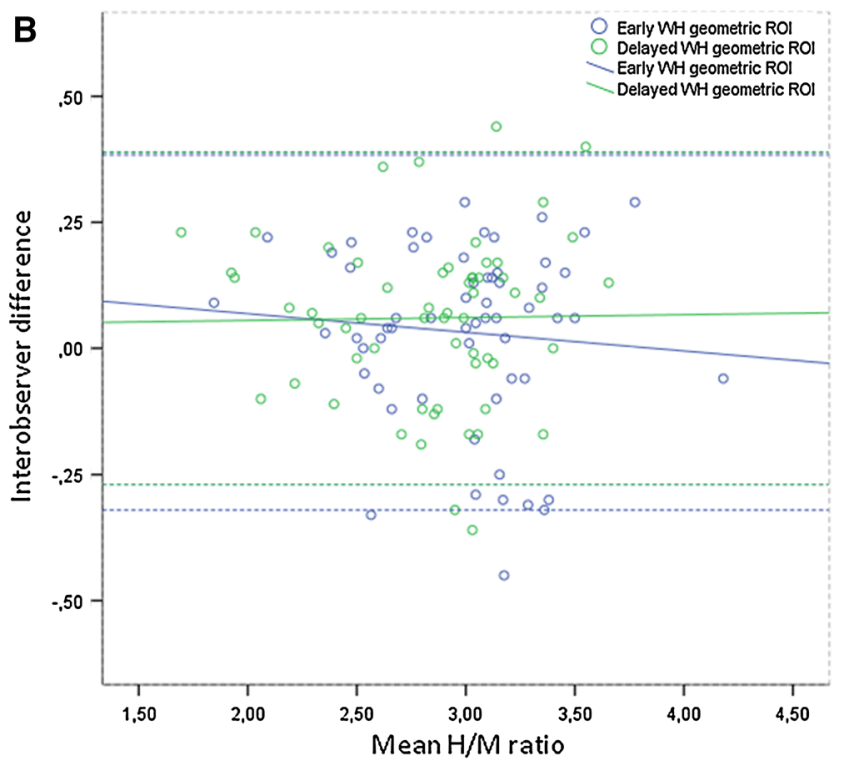

$0.01), R^{2}=1.0 \mathrm{e}^{-5}$; delayed images -0.01 (95\% CI -0.07 to 0.04$)$, $R^{2}=0.01$. B. Mean differences: early images $0.03(95 \% \mathrm{CI}-0.01$ to $0.08) R^{2}=0.01$; delayed images $0.06(95 \%$ CI $0.02-0.10), R^{2}=2.2$ $\mathrm{e}^{-4}$

Table 3 IRW-derived ${ }^{123}$ I- $m$ IBG SPECT indices

\begin{tabular}{lll}
\hline & \multicolumn{2}{l}{ Mean (SD) } \\
\cline { 2 - 3 } & WH & LV \\
\hline Early H/M ratio & $4.28(0.96)$ & $4.39(0.99)^{\dagger}$ \\
Delayed H/M ratio & $4.35(1.24)$ & $4.45(1.27)^{\dagger}$ \\
Washout rate (\%) & $20.9(14.9)$ & $21.5(14.4)$ \\
\hline
\end{tabular}

$W H$ whole-heart ROI, $L V$ left ventricle ROI

$\dagger p<0.001$

\section{Multivariate analysis}

Since delayed anterior $\mathrm{WH} \mathrm{H} / \mathrm{M}$ ratio seems to be the most robust measurement in our population, we aimed to identify by which parameters it was influenced. Multivariate analysis showed that GRS was the only independent predictor of the $\mathrm{WH} \mathrm{H} / \mathrm{M}$ ratio (standardized $\beta=0.36, p=0.023$ ).

\section{Discussion}

In the current study we examined the interrelationship of an extensive panel of potential parameters for the early detection of chronic AIC, in a homogenous group of breast cancer survivors 1 year after treatment. There was a significant correlation between the delayed planar $\mathrm{WH} \mathrm{H} / \mathrm{M}$ ratio and several conventional echocardiographic values, GLS, 


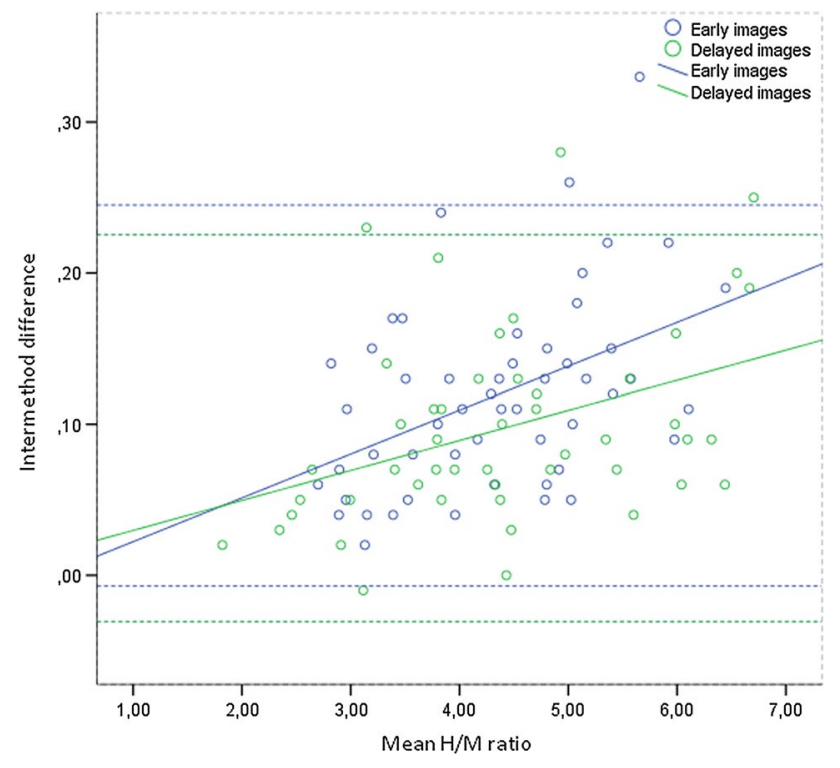

Fig. 4 IRW-derived ${ }^{123}$ I- $m$ IBG SPECT intermethod differences. Early WH versus LV H/M ratio: mean difference 0.12 , LCC 0.99 (95 \% CI 0.985-0.994), $R^{2}=0.20$. Delayed WH versus LV H/M ratio: mean difference 0.10, LCC 0.996 (95 \% CI 0.993-0.997), $R^{2}=0.15$. Butted lines represent $95 \%$ limits of agreement. The differences can be accounted for by the dilution effect. WO mean difference $-0.5 \%$, LCC 0.993 (95\% CI 0.987-0.996), not included in figure

GRS and galectin-3. Furthermore, GRS was identified as an independent predictor of the late planar $\mathrm{WH} \mathrm{H/M}$ ratio.

Because high reproducibility is an important requirement for any diagnostic modality, we first evaluated the interobserver and intermethod variability of different methods of $\mathrm{H} / \mathrm{M}$ ratio calculation to identify the most robust one, since the delineation method is a main factor hampering widespread clinical use of cardiac ${ }^{123} \mathrm{I}-m \mathrm{IBG}$ scintigraphy $[10,14,35]$. In most clinical studies, the WH ROI on anterior planar images is used [2, 6, 20,33]. We showed that indices based on the geometric mean based did not improve reproducibility, nor was there a difference between early and delayed indices. Although a Sm ROI reduces a possible 'dilution effect' (Fig. 3), it is inferior to the WH ROI because of its high observer variability (Table 2). The Sm ROI should therefore not be used.

The addition of SPECT to ${ }^{123} \mathrm{I}-m \mathrm{IBG}$ scintigraphy might increase the diagnostic potential of this technique. Although most studies focus on regional sympathetic innervation rather than a global SPECT assessment, Chen et al. [13] described a high reproducibility and accuracy of global ${ }^{123}$ I- $m$ IBG SPECT evaluation, which allows to separate heart failure patients from healthy controls. We observed a high correlation between planar and SPECTderived parameters, especially for WO. Furthermore, SPECT H/M ratios were systematically higher than planar ratios, which is most likely caused by an overestimation of background activity on planar images [13]. Although SPECT reconstructions result in a more accurate calculation of the H/M ratio, SPECT image acquisition and reconstruction are time-consuming and performed with different protocols, yielding divergent $\mathrm{H} / \mathrm{M}$ ratio ranges, thus hindering standardization [13]. Hence, global ${ }^{123} \mathrm{I}-m$ IBG SPECT imaging, although promising, does not provide sufficient added value to be recommended for use in daily clinical practice.

Several conventional echocardiographic parameters, GLS, GRS and galectin-3 showed a correlation with WH $\mathrm{H} / \mathrm{M}$ ratio, but only GRS proved to be an independent predictor.

GRS measures the relative deformation of the cardiac left ventricular wall in the radial direction (i.e. LV wall thickening), yielding a positive strain value during systole. In AIC, functioning myocytes are replaced by non-contracting fibrotic cells [32], which leads to impaired cardiac thickening and a decrease in GRS. Due to the sympathetic response to myocardial damage, the $\mathrm{H} / \mathrm{M}$ ratio will also decrease. Therefore, theoretically one would expect a direct relationship between GRS and WH H/M ratio, which was confirmed by the results of our study. Since GLS measures shortening of the myocardial wall in the longitudinal axis, it is defined as a negative value and an inverse relationship with WH H/M ratio is expected. The results of our study indeed demonstrate an inverse correlation between GLS and $\mathrm{WH} \mathrm{H} / \mathrm{M}$ ratio, although not strong enough to predict the $\mathrm{WH} \mathrm{H} / \mathrm{M}$ ratio. No correlation, however, was observed between GCS and WH H/M ratio.

Of the studied biomarkers, only the novel blood biomarker galectin-3 showed a significant correlation with the WH H/M ratio. Galectin-3 is a protein expressed by macrophages and believed to be a mediator of the profibrotic pathway, stimulating cardiac fibroblasts to proliferate and deposit collagen [22]. Galectin-3 concentrations are elevated in patients with acute HF and predict an adverse outcome [29]. In a recent study by Ky et al., no association between cardiotoxicity and galectin-3 was found, although follow-up only lasted 6 months [22]. Other studies on this issue have not been performed in adults, but a recent study in paediatric patients showed an (non-significantly) increased level of galectin-3 at least 2 years after anthracycline treatment [5].

Conventional echocardiographic parameters that correlated with the WH H/M ratio included LVEDV/BSA, LVESV/BSA, IVSd, LVM/BSA and E/A ratio. The correlation of the WH H/M ratio and LVEDV, LVESV, IVSd and LVM displayed an inverse nature, which means that both cardiac volumes and cardiac wall diameters increase as the WH H/M ratio drops. This is an interesting finding, since typical AIC in adults presents as a dilated cardiomyopathy, 
Table 4 Conventional and strain (rate) echocardiography and biomarker results

\begin{tabular}{|c|c|c|c|c|c|}
\hline & \multicolumn{2}{|c|}{ Study population } & \multirow[t]{2}{*}{ Reference value (RV) } & \multicolumn{2}{|c|}{ Abnormal values $^{\mathrm{a}}$} \\
\hline & $N$ & Mean (SD) & & $<\mathrm{RV}$ & $>\mathrm{RV}$ \\
\hline \multicolumn{6}{|c|}{ Conventional echocardiography } \\
\hline $\mathrm{EF}(\%)$ & 59 & $62.6(7.1)$ & $\geq 55$ & $4(7)$ & N/A \\
\hline LVEDV/BSA $\left(\mathrm{ml} / \mathrm{m}^{2}\right)$ & 58 & $46.2(10.0)$ & $35-75$ & $7(12)$ & $1(2)$ \\
\hline LVESV/BSA $\left(\mathrm{ml} / \mathrm{m}^{2}\right)$ & 58 & $18.0(6.3)$ & $12-30$ & $9(16)$ & $1(2)$ \\
\hline LVIDd (cm) & 59 & $4.6(0.5)$ & $3.9-5.3$ & $3(5)$ & $7(12)$ \\
\hline $\operatorname{IVSd}(\mathrm{cm})$ & 59 & $0.9(0.1)$ & $0.6-0.9$ & 0 & $14(24)$ \\
\hline LVPWd (cm) & 59 & $0.9(0.1)$ & $0.6-0.9$ & 0 & $15(25)$ \\
\hline $\operatorname{LVM} / \mathrm{BSA}\left(\mathrm{g} / \mathrm{m}^{2}\right)$ & 59 & 77 (17) & $44-88$ & 0 & $12(20)$ \\
\hline LAEDV/BSA $\left(\mathrm{ml} / \mathrm{m}^{2}\right)^{\mathrm{a}}$ & 57 & $22(6)$ & $22( \pm 6)$ & $1(2)$ & $2(4)$ \\
\hline $\mathrm{E} / \mathrm{e}^{\prime}$ ratio & 52 & $6.5(2.3)$ & $<8$ & N/A & $10(19)$ \\
\hline E/A ratio & 58 & $1.2(0.3)$ & $1.3( \pm 0.3)$ & $3(6)$ & $3(6)$ \\
\hline PV S/D ratio & 54 & $1.4(0.4)$ & $1.2( \pm 0.2)$ & $3(6)$ & $15(28)$ \\
\hline \multicolumn{6}{|c|}{$2 D$ strain (rate) echocardiography } \\
\hline GLS & 57 & $-17.7(3.1)$ & $-17.8(2.1)$ & $2(4)$ & $6(11)$ \\
\hline GLSR & 57 & $-0.87(0.2)$ & $-0.87(0.1)$ & $3(5)$ & $2(4)$ \\
\hline GRS & 42 & $38.0(10.0)$ & $40.5(11.4)$ & $1(2)$ & 0 \\
\hline GRSR & 41 & $1.45(0.4)$ & $2.20(0.6)$ & 0 & 0 \\
\hline GCS & 42 & $-18.9(4.6)$ & $-20.3(2.6)$ & $3(7)$ & $7(17)$ \\
\hline GCSR & 41 & $-1.03(0.2)$ & $-1.72(0.3)$ & 0 & $24(57)$ \\
\hline \multicolumn{6}{|c|}{ Median (interquartile range) } \\
\hline \multicolumn{6}{|l|}{ Biomarkers } \\
\hline NT-proBNP (pg/ml) & 59 & $119(127)$ & $\begin{array}{l}18-50 \text { years: }<170 \\
50-60 \text { years: }<250 \\
60-70 \text { years: }<300^{\dagger}\end{array}$ & N/A & $10(17)$ \\
\hline Troponin I ( $\mu \mathrm{g} / \mathrm{l})$ & 59 & $<0.2(<0.2)$ & $<0.2^{\dagger}$ & N/A & $0(0)$ \\
\hline TNF- $\alpha(\mathrm{pg} / \mathrm{ml})$ & 55 & $<2.8(1.5)$ & $<10^{\ddagger}$ & N/A & $4(7)$ \\
\hline Galectin-3 (ng/ml) & 55 & $12.9(3.6)$ & $<17.6^{\ddagger}$ & N/A & $4(7)$ \\
\hline IL-6 (pg/ml) & 55 & $<3.12(<3.12)$ & $<10^{\ddagger}$ & N/A & $0(0)$ \\
\hline ST2 (ng/ml) & 55 & $10.6(14.4)$ & $4.9-19.9^{\ddagger}$ & $0(0)$ & $0(0)$ \\
\hline sFlt-1 (pg/ml) & 55 & $<320(<320)$ & Not detectable & N/A & $0(0)$ \\
\hline
\end{tabular}

$E F$ ejection fraction, $L V E D V$ left ventricular end-diastolic volume, $B S A$ body surface area, $L V E S V$ left ventricular end-systolic volume, LVIDd left ventricle internal dimension at diastole, IVSd interventricular septum at diastole, $L V P W d$ left ventricular posterior wall at diastole, $L V M$ left ventricular mass, $L A E D V$ left atrial end-diastolic volume, E/e' ratio early diastolic transmitral peak flow velocity (E) to early diastolic annular velocity ( $\mathrm{e}^{\prime}$ ) ratio, E/A ratio early (E) and late (A) diastolic transmitral peak flow velocity ratio, $S / D$ ratio systolic/diastolic ratio, GLS global longitudinal strain, GLSR global longitudinal strain rate, GRS global radial strain, GRSR global radial strain rate, GCS global circumferential strain, GCSR global circumferential strain rate, $N / A$ not applicable

$\dagger \mathrm{RV}$ as established by the Radboudumc chemical laboratory

$¥ \mathrm{RV}$ as stated in manufacturer's protocol

a Abnormal value is outside $2 \mathrm{SD}$ when reference is defined as a mean $\pm \mathrm{SD}$ featuring increased ventricle sizes and thin walls [18]. A possible explanation for this increased wall diameter is a compensatory myocyte hypertrophy in the dilated heart. This pattern has been described before in childhood cancer survivors [5, 23]. Another interesting finding is that the relative deformation (represented by the GRS and GLS) decreases, while the cardiac wall diameter increases. This probably reflects the replacement of active myocytes by passive (fibrotic) tissue and concurrent myocyte hypertrophy. Furthermore, we observed a (weak) direct correlation of the E/A ratio with the WH H/M ratio, indicating a concurrent decrease in the E/A ratio with the WH H/M ratio. The E velocity indicates diastolic filling, which decreases gradually in normal subjects. The A velocity reflects the 
Table 5 Overview of interrelationship of different parameters

\begin{tabular}{|c|c|c|c|c|c|c|c|c|c|c|c|c|c|c|c|c|c|c|c|}
\hline & & \multicolumn{3}{|c|}{${ }^{123}$ I-mIBG scintigraphy } & \multicolumn{6}{|c|}{ Conventional echo } & \multicolumn{6}{|c|}{ Strain echo } & \multicolumn{3}{|c|}{ Biomarkers } \\
\hline & & 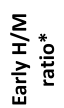 & 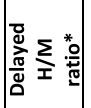 & $\stackrel{*}{3}$ & 岀 & 总 & 岕 & 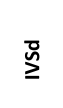 & $\sum$ & $\begin{array}{l}\stackrel{ }{0} \\
\frac{\pi}{\pi} \\
\frac{1}{W}\end{array}$ & ปั & 站 & 参 & $\begin{array}{l}\text { 产 } \\
\text { ญิ }\end{array}$ & પ્ડ & 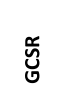 & 毫高 & $\begin{array}{l}\frac{\pi}{\pi} \\
\frac{1}{4} \\
1\end{array}$ & 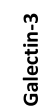 \\
\hline \multirow[t]{2}{*}{$\begin{array}{l}{ }^{123} \text { I-mIBG } \\
\text { scintigraphy }\end{array}$} & $\begin{array}{l}\text { Delayed } \\
\text { H/M ratio* }\end{array}$ & 0.80 & $x$ & $x$ & $x$ & $x$ & $x$ & $x$ & $x$ & $x$ & $x$ & $x$ & $x$ & $x$ & $x$ & $x$ & $x$ & $x$ & $x$ \\
\hline & wo* & -0.08 & -0.53 & $x$ & $x$ & $x$ & $x$ & $x$ & $x$ & $x$ & $x$ & $x$ & $x$ & $x$ & $x$ & $x$ & $x$ & $x$ & $x$ \\
\hline \multirow{6}{*}{$\begin{array}{c}\text { Conventional } \\
\text { echo }\end{array}$} & $\mathrm{EF}$ & -0.12 & 0.034 & -0.28 & $x$ & $x$ & $x$ & $x$ & $x$ & $x$ & $x$ & $x$ & $x$ & $x$ & $x$ & $x$ & $x$ & $x$ & $x$ \\
\hline & LVEDV & -0.18 & -0.34 & 0.23 & -0.34 & $x$ & $x$ & $x$ & $x$ & $x$ & $x$ & $x$ & $x$ & $x$ & $x$ & $x$ & $x$ & $x$ & $x$ \\
\hline & LVESV & -0.08 & -0.24 & 0.27 & -0.75 & 0.82 & $x$ & $x$ & $x$ & $x$ & $x$ & $x$ & $x$ & $x$ & $x$ & $x$ & $x$ & $x$ & $x$ \\
\hline & IVSd & -0.23 & -0.27 & 0.19 & -0.04 & 0.09 & 0.05 & $x$ & $\mathrm{x}$ & $\mathrm{X}$ & $\mathrm{x}$ & $\mathrm{X}$ & $\mathrm{x}$ & $\mathrm{x}$ & $\mathrm{x}$ & $\mathrm{x}$ & $\mathrm{x}$ & $\mathrm{X}$ & $x$ \\
\hline & LVM & -0.18 & -0.23 & 0.23 & -0.22 & 0.19 & 0.29 & 0.58 & $x$ & $x$ & $x$ & $x$ & $x$ & $x$ & $x$ & $x$ & $x$ & $x$ & $x$ \\
\hline & E/A ratio & 0.15 & -0.19 & -0.17 & 0.03 & -0.24 & -0.19 & -0.16 & -0.28 & $\mathrm{x}$ & $x$ & $x$ & $x$ & $\mathrm{x}$ & $x$ & $x$ & $x$ & $\mathrm{x}$ & $x$ \\
\hline \multirow{6}{*}{ Strain echo } & GLS & -0.09 & -0.23 & 0.33 & -0.39 & 0.10 & 0.35 & -0.03 & 0.40 & -0.44 & $x$ & $\mathrm{x}$ & $x$ & $x$ & $x$ & $x$ & $x$ & $\mathrm{x}$ & $x$ \\
\hline & GLSR & -0.01 & -0.11 & 0.19 & -0.39 & 0.18 & 0.38 & -0.13 & 0.25 & 0.05 & 0.65 & $\mathrm{x}$ & $\mathrm{x}$ & $x$ & $x$ & $x$ & $x$ & $x$ & $x$ \\
\hline & GRS & 0.23 & 0.36 & -0.19 & 0.24 & -0.39 & -0.42 & -0.17 & -0.49 & 0.49 & -0.36 & -0.17 & $x$ & $x$ & $x$ & $x$ & $x$ & $\mathrm{X}$ & $x$ \\
\hline & GRSR & -0.09 & 0.07 & -0.04 & -0.06 & -0.11 & -0.05 & -0.03 & -0.34 & 0.12 & -0.11 & -0.15 & 0.37 & $x$ & $x$ & $x$ & $x$ & $x$ & $x$ \\
\hline & GCS & -0.04 & -0.03 & -0.11 & -0.36 & 0.23 & 0.38 & -0.31 & 0.09 & -0.24 & 0.31 & 0.17 & -0.18 & -0.05 & $x$ & $x$ & $x$ & $x$ & $x$ \\
\hline & GCSR & 0.02 & -0.16 & 0.12 & -0.30 & 0.25 & 0.35 & -0.18 & 0.28 & -0.16 & 0.35 & 0.44 & -0.33 & -0.61 & 0.51 & $x$ & $x$ & $x$ & $x$ \\
\hline \multirow{4}{*}{ Biomarkers } & NT-proBNP & -0.00 & -0.16 & 0.23 & -0.56 & 0.38 & 0.65 & 0.12 & 0.36 & -0.17 & 0.44 & 0.38 & -0.25 & -0.08 & 0.41 & 0.42 & $x$ & $x$ & $x$ \\
\hline & TNF-alfa & 0.19 & 0.16 & 0.04 & -0.14 & 0.21 & 0.20 & -0.14 & 0.07 & -0.08 & 0.03 & -0.16 & -0.05 & -0.18 & 0.09 & 0.07 & -0.07 & $x$ & $x$ \\
\hline & Galectin-3 & -0.13 & -0.30 & 0.09 & 0.03 & 0.17 & 0.06 & 0.15 & 0.09 & -0.32 & 0.11 & -0.05 & -0.14 & 0.04 & 0.08 & 0.12 & 0.01 & 0.20 & $x$ \\
\hline & ST2 & -0.01 & -0.05 & 0.01 & 0.03 & -0.22 & -0.18 & -0.06 & -0.11 & -0.10 & 0.16 & 0.11 & -0.13 & -0.27 & 0.21 & 0.33 & -0.06 & -0.10 & 0.19 \\
\hline
\end{tabular}

* Planar anterior WH region. Green $p<0.01$. Blue $p<0.05 . \mathrm{H} / \mathrm{M}$ ratio $=$ heart/mediastinum ratio; WO washout, EF ejection fraction, GLS global longitudinal strain, GLSR global longitudinal strain rate, GRS global radial strain, GRSR global radial strain rate, GCS global circumferential strain, GCSR global circumferential strain rate, $L V E D V$ left ventricular end-diastolic volume, $L V E S V$ left ventricular end-systolic volume, $I V S d$ interventricular septum at diastole, $L V M$ left ventricular mass, E/A ratio early (E) and late (A) diastolic transmitral peak flow velocity ratio. LVEDV, LVESV and LVM are indexed by body surface area. Troponin, IL-6 and sFlt-1 have not been tested because there were no abnormal results

active atrial contraction just before end-diastole and normally becomes more important in elderly patients, resulting in an E/A ratio approaching 1 [8]. However, a decreased E/A ratio could also imply diastolic dysfunction, indicating AIC, although opinions on the usefulness of the E/A ratio differ [4, 28].

The main limitation of the current study is the lack of baseline and follow-up measurements, so we could not assess the change in parameters over time. However, the aim of the study was not to detect a change in certain parameters, nor to predict AIC, but to assess the interrelationship of different interesting parameters in the pathophysiological process of AIC.

Although the correlations of certain parameters have been studied, for example strain (rate) imaging and troponin or conventional echocardiography and strain (rate) imaging, they have not been studied for their correlation with ${ }^{123}$ I- $m$ IBG parameters. Furthermore, we focused on a homogenous group of breast carcinoma survivors with potential AIC damage, which is a patient group that has not yet been studied properly.

With the current study we have identified the relationship of the $\mathrm{WH} \mathrm{H} / \mathrm{M}$ ratio with $2 \mathrm{D}$ strain imaging, biomarkers and conventional echocardiography 1 year after anthracycline-based chemotherapy. This sheds some light on the complex pathophysiology of AIC, enabling future studies to identify appropriate parameters for the detection of AIC.

\section{Conclusions}

Delayed planar WH H/M ratio is the most robust ${ }^{123} \mathrm{I}-m \mathrm{IBG}$ parameter. It is correlated with several conventional echocardiographic parameters, GLS, GRS and galectin-3. Of these, only GRS is an independent predictor of the WH H/M ratio. Future studies should concentrate on a combination of ${ }^{123}$ I- $m$ IBG scintigraphy, MUGA, echocardiographic strain, CMR and biomarkers, preferably in a prospective multicentre trial with long-term follow-up in breast cancer survivors.

Acknowledgments We kindly thank Caroline Mandigers and Harald Fliervoet for contributing to the enrolment of patients in our study. We thank Wim van Boxtel for his help in questionnaire processing.

Funding This study was financially supported by Cephalon B.V., Merck Sharp \& Dohme B.V. and Sanofi-Aventis Netherlands B.V.

\section{Compliance with ethical standards}

Conflict of interest The study sponsors had no involvement in the study.

Open Access This article is distributed under the terms of the Creative Commons Attribution 4.0 International License (http://creativecommons.org/licenses/by/4.0/), which permits unrestricted use, distribution, and reproduction in any medium, provided you give appropriate credit to the original author(s) and the source, provide a link to the Creative Commons license, and indicate if changes were made. 


\section{References}

1. Aggarwal A, Esler MD, Socratous F, Kaye DM (2001) Evidence for functional presynaptic alpha-2 adrenoceptors and their down-regulation in human heart failure. J Am Coll Cardiol 37:1246-1251

2. Agostini D, Verberne HJ, Burchert W, Knuuti J, Povinec P, Sambuceti G, Unlu M, Estorch M, Banerjee G, Jacobson AF (2008) I-123-mIBG myocardial imaging for assessment of risk for a major cardiac event in heart failure patients: insights from a retrospective European multicenter study. Eur J Nucl Med Mol Imaging 35:535-546

3. Altena R, Perik PJ, van Veldhuisen DJ, de Vries EG, Gietema JA (2009) Cardiovascular toxicity caused by cancer treatment: strategies for early detection. Lancet Oncol 10:391-399

4. Appel JM, Sogaard P, Mortensen CE, Skagen K, Nielsen DL (2011) Tissue-Doppler assessment of cardiac left ventricular function during short-term adjuvant epirubicin therapy for breast cancer. J Am Soc Echocardiogr 24:200-206

5. Armenian SH, Gelehrter SK, Vase T, Venkatramani R, Landier W, Wilson KD, Herrera C, Reichman L, Menteer JD, Mascarenhas L, Freyer DR, Venkataraman K, Bhatia S (2014) Screening for cardiac dysfunction in anthracycline-exposed childhood cancer survivors. Clin Cancer Res 20:6314-6323

6. Boogers MJ, Borleffs CJ, Henneman MM, van Bommel RJ, van Ramshorst J, Boersma E, Dibbets-Schneider P, Stokkel MP, van der Wall EE, Schalij MJ, Bax JJ (2010) Cardiac sympathetic denervation assessed with 123-iodine metaiodobenzylguanidine imaging predicts ventricular arrhythmias in implantable cardioverter-defibrillator patients. J Am Coll Cardiol 55:2769-2777

7. Braunwald E (2008) Biomarkers in heart failure. N Engl J Med 358:2148-2159

8. Bryg RJ, Williams GA, Labovitz AJ (1987) Effect of aging on left ventricular diastolic filling in normal subjects. Am J Cardiol 59:971-974

9. Bulten BF, Mavinkurve-Groothuis AM, de Geus-Oei LF, de Haan AF, de Korte CL, Bellersen L, van Laarhoven HW, Kapusta L (2014) Early myocardial deformation abnormalities in breast cancer survivors. Breast Cancer Res Treat 146:127-135

10. Bulten BF, van der Palen RL, van Laarhoven HW, Kapusta L, Mavinkurve-Groothuis AM, de Geus-Oei LF (2012) Interobserver variability of heart-to-mediastinum ratio in I-123 MIBG sympathetic imaging. Curr Cardiol Rep 14:389-390 (author reply 391)

11. Cardoso F, Costa A, Norton L, Senkus E, Aapro M, Andre F, Barrios $\mathrm{CH}$, Bergh J, Biganzoli L, Blackwell KL, Cardoso MJ, Cufer T, El Saghir N, Fallowfield L, Fenech D, Francis P, Gelmon K, Giordano SH, Gligorov J, Goldhirsch A, Harbeck N, Houssami N, Hudis C, Kaufman B, Krop I, Kyriakides S, Lin UN, Mayer M, Merjaver SD, Nordstrom EB, Pagani O, Partridge A, Penault-Llorca F, Piccart MJ, Rugo H, Sledge G, Thomssen C, Van't Veer L, Vorobiof D, Vrieling C, West N, Xu B, Winer E (2014) ESO-ESMO 2nd international consensus guidelines for advanced breast cancer (ABC2). Breast 23:489-502

12. Carrio I (2001) Cardiac neurotransmission imaging. J Nucl Med 42:1062-1076

13. Chen J, Folks RD, Verdes L, Manatunga DN, Jacobson AF, Garcia EV (2012) Quantitative I-123 mIBG SPECT in differentiating abnormal and normal mIBG myocardial uptake. J Nucl Cardiol 19:92-99

14. Chen W, Cao Q, Dilsizian V (2011) Variation of heart-to-mediastinal ratio in (123)I-mIBG cardiac sympathetic imaging: its affecting factors and potential corrections. Curr Cardiol Rep 13:132-137

15. Chirumamilla A, Travin MI (2011) Cardiac applications of 123I-mIBG imaging. Semin Nucl Med 41:374-387
16. Flotats A, Carrio I, Agostini D, Le Guludec D, Marcassa C, Schafers M, Somsen GA, Unlu M, Verberne HJ (2010) Proposal for standardization of 123I-metaiodobenzylguanidine (MIBG) cardiac sympathetic imaging by the EANM Cardiovascular Committee and the European Council of Nuclear Cardiology. Eur J Nucl Med Mol Imaging 37:1802-1812

17. Gianni L, Herman EH, Lipshultz SE, Minotti G, Sarvazyan N, Sawyer DB (2008) Anthracycline cardiotoxicity: from bench to bedside. J Clin Oncol 26:3777-3784

18. Giantris A, Abdurrahman L, Hinkle A, Asselin B, Lipshultz SE (1998) Anthracycline-induced cardiotoxicity in children and young adults. Crit Rev Oncol Hematol 27:53-68

19. Gutierrez-Chico JL, Zamorano JL, Perez de Isla L, Orejas M, Almeria C, Rodrigo JL, Ferreiros J, Serra V, Macaya C (2005) Comparison of left ventricular volumes and ejection fractions measured by three-dimensional echocardiography versus by twodimensional echocardiography and cardiac magnetic resonance in patients with various cardiomyopathies. Am J Cardiol 95:809-813

20. Jacobson AF, Senior R, Cerqueira MD, Wong ND, Thomas GS, Lopez VA, Agostini D, Weiland F, Chandna H, Narula J (2010) Myocardial iodine-123 meta-iodobenzylguanidine imaging and cardiac events in heart failure. Results of the prospective ADMIRE-HF (AdreView Myocardial Imaging for Risk Evaluation in Heart Failure) study. J Am Coll Cardiol 55:2212-2221

21. Ky B, French B, Ruparel K, Sweitzer NK, Fang JC, Levy WC, Sawyer DB, Cappola TP (2011) The vascular marker soluble fms-like tyrosine kinase 1 is associated with disease severity and adverse outcomes in chronic heart failure. J Am Coll Cardiol 58:386-394

22. Ky B, Putt M, Sawaya H, French B, Januzzi JL Jr, Sebag IA, Plana JC, Cohen V, Banchs J, Carver JR, Wiegers SE, Martin RP, Picard MH, Gerszten RE, Halpern EF, Passeri J, Kuter I, Scherrer-Crosbie M (2014) Early increases in multiple biomarkers predict subsequent cardiotoxicity in patients with breast cancer treated with doxorubicin, taxanes, and trastuzumab. J Am Coll Cardiol 63:809-816

23. Lipshultz SE, Lipsitz SR, Sallan SE, Dalton VM, Mone SM, Gelber RD, Colan SD (2005) Chronic progressive cardiac dysfunction years after doxorubicin therapy for childhood acute lymphoblastic leukemia. J Clin Oncol 23:2629-2636

24. Lyu YL, Kerrigan JE, Lin CP, Azarova AM, Tsai YC, Ban Y, Liu LF (2007) Topoisomerase IIbeta mediated DNA double-strand breaks: implications in doxorubicin cardiotoxicity and prevention by dexrazoxane. Cancer Res 67:8839-8846

25. Marwick TH (2006) Measurement of strain and strain rate by echocardiography: ready for prime time? J Am Coll Cardiol 47:1313-1327

26. McBride GB (2005) A proposal for strength-of-agreement criteria for Lin's concordance correlation coefficient. In: MO Health (ed) National Institute of Water \& Atmospheric Research Ltd. Hamilton, New Zealand, pp 1-10

27. Partridge AH, Rumble RB, Carey LA, Come SE, Davidson NE, Di Leo A, Gralow J, Hortobagyi GN, Moy B, Yee D, Brundage SB, Danso MA, Wilcox M, Smith IE (2014) Chemotherapy and targeted therapy for women with human epidermal growth factor receptor 2-negative (or unknown) advanced breast cancer: American Society of Clinical Oncology clinical practice guideline. J Clin Oncol 32:3307-3329

28. Radulescu D, Pripon S, Radulescu LI, Duncea C (2008) Left ventricular diastolic performance in breast cancer survivors treated with anthracyclines. Acta Cardiol 63:27-32

29. Shah RV, Chen-Tournoux AA, Picard MH, van Kimmenade RR, Januzzi JL (2010) Galectin-3, cardiac structure and function, and long-term mortality in patients with acutely decompensated heart failure. Eur J Heart Fail 12:826-832 
30. Somsen GA, Verberne HJ, Fleury E, Righetti A (2004) Normal values and within-subject variability of cardiac I-123 MIBG scintigraphy in healthy individuals: implications for clinical studies. J Nucl Cardiol 11:126-133

31. Stoodley PW, Richards DA, Hui R, Boyd A, Harnett PR, Meikle SR, Clarke J, Thomas L (2011) Two-dimensional myocardial strain imaging detects changes in left ventricular systolic function immediately after anthracycline chemotherapy. Eur J Echocardiogr 12:945-952

32. Takemura G, Fujiwara H (2007) Doxorubicin-induced cardiomyopathy from the cardiotoxic mechanisms to management. Prog Cardiovasc Dis 49:330-352

33. Tamaki S, Yamada T, Okuyama Y, Morita T, Sanada S, Tsukamoto Y, Masuda M, Okuda K, Iwasaki Y, Yasui T, Hori M, Fukunami M (2009) Cardiac iodine-123 metaiodobenzylguanidine imaging predicts sudden cardiac death independently of left ventricular ejection fraction in patients with chronic heart failure and left ventricular systolic dysfunction: results from a comparative study with signal-averaged electrocardiogram, heart rate variability, and QT dispersion. J Am Coll Cardiol 53:426-435
34. van Boxtel W, Bulten BF, Mavinkurve-Groothuis AM, Bellersen L, Mandigers CM, Joosten LA, Kapusta L, de Geus-Oei LF, van Laarhoven HW (2015) New biomarkers for early detection of cardiotoxicity after treatment with docetaxel, doxorubicin, and cyclophosphamide. Biomarkers 20(2):143-148. doi:10.3109/135 4750X.2015.1040839

35. Veltman CE, Boogers MJ, Meinardi JE, Al Younis I, DibbetsSchneider P, Van der Wall EE, Bax JJ, Scholte AJ (2012) Reproducibility of planar (123)I-meta-iodobenzylguanidine (MIBG) myocardial scintigraphy in patients with heart failure. Eur J Nucl Med Mol Imaging 39:1599-1608

36. Wood PW, Choy JB, Nanda NC, Becher H (2014) Left ventricular ejection fraction and volumes: it depends on the imaging method. Echocardiography 31:87-100

37. Yeh ET, Bickford CL (2009) Cardiovascular complications of cancer therapy: incidence, pathogenesis, diagnosis, and management. J Am Coll Cardiol 53:2231-2247

38. Zhang S, Liu X, Bawa-Khalfe T, Lu LS, Lyu YL, Liu LF, Yeh ET (2012) Identification of the molecular basis of doxorubicininduced cardiotoxicity. Nat Med 18:1639-1642 